\title{
Multigene Test
}

National Cancer Institute ( $\mathrm{NCl})$

\section{Source}

National Cancer Institute (NCI). Multigene Test.

Genetic tests that use next-generation sequencing to test multiple genes simultaneously. Also called multiple-gene panel test and multiple-gene test. 